\title{
Specific Expression of Interferon- $\gamma$ Induced by Synergistic Activation Mediator-Derived Systems Activates Innate Immunity and Inhibits Tumorigenesis ${ }^{\text {s }}$
}

\author{
Shuai Liu ${ }^{1}$, Xiao $\mathrm{Yu}^{2}$, Qiankun Wang ${ }^{1}$, Zhepeng $\mathrm{Liu}^{1}$, Qiaoqiao Xiao ${ }^{1}$, Panpan $\mathrm{Hou}^{3}$, Ying $\mathrm{Hu}^{3}$, Wei Hou ${ }^{1}$, \\ Zhanqiu Yang ${ }^{1}$, Deyin Guo ${ }^{1,4 *}$, and Shuliang Chen ${ }^{1,5 *}$ \\ ${ }^{1}$ School of Basic Medical Sciences, Wuhan University, Wuhan 430071, P.R. China \\ ${ }^{2}$ Institute for Infectious Disease Control and Prevention, Hubei Provincial Center for Disease Control and Prevention, Wuhan 430079, P.R. China \\ ${ }^{3}$ College of Life Sciences, Wuhan University, Wuhan 430072, P.R. China \\ ${ }^{4}$ School of Medicine (Shenzhen), Sun Yat-sen University, Guangzhou 510080, P.R. China \\ ${ }^{5}$ Center for Retrovirus Research, Department of Veterinary Biosciences, The Ohio State University, Columbus, OH 43210, USA
}

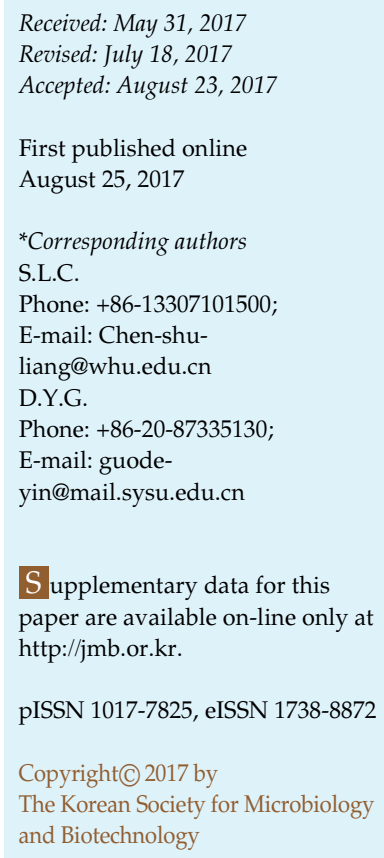

The synergistic activation mediator (SAM) system can robustly activate endogenous gene expression by a single-guide RNA. This transcriptional modulation has been shown to enhance gene promoter activity and leads to epigenetic changes. Human interferon- $\gamma$ is a common natural glycoprotein involved in antiviral effects and inhibition of cancer cell growth. Large quantities of high-purity interferon- $\gamma$ are important for medical research and clinical therapy. To investigate the possibility of employing the SAM system to enhance endogenous human interferon- $\gamma$ with normal function in innate immunity, we designed 10 single-guide RNAs that target 200 bp upstream of the transcription start sites of the interferon- $\gamma$ genome, which could significantly activate the interferon- $\gamma$ promoter reporter. We confirmed that the system can effectively and highly activate interferon- $\gamma$ expression in several humanized cell lines. Moreover, we found that the interferon- $\gamma$ induced by the SAM system could inhibit tumorigenesis. Taken together, our results reveal that the SAM system can modulate epigenetic traits of non-immune cells through activating interferon- $\gamma$ expression and triggering JAK-STAT signaling pathways. Thus, this strategy could offer a novel approach to inhibit tumorigenesis without using exogenous interferon- $\gamma$.

Keywords: Synergistic activation mediator (SAM), CRISPR-dCas9, humanized interferon- $\gamma$, JAK-STAT signaling pathway, tumorigenesis

\section{Introduction}

A plethora of clinical studies have extensively documented that interferon- $\gamma$ has antiviral effects mainly by acting on macrophages and T-lymphocytes to regulate innate immunity. $\mathrm{T}$ cells are important for protection against intracellular pathogens, as they are involved in the production of macrophage-activating factors, such as interferon- $\gamma$ [1]. These highly active glycoproteins are expressed only in response to pathogens or microbial stimulation and constitute the main barrier to virus infection [2]. Current studies have suggested that interferon- $\gamma$ also inhibits the proliferation of cancer cells in vitro and induces apoptosis through adopting different mechanisms [3, 4]. Given the significant roles in medical research and clinical treatment, there has been substantial interest in exploring optimal approaches to acquire highly purified interferon- $\gamma$. Some previous research reported feasible methods to express interferon- $\gamma$, such as employing adenovirus, Escherichia coli (E. coli) [5, 6], Saccharomyces cerevisiae [7], Pichia pastoris [8], baculovirusinsect cell equipped double cistrons [9], and Chinese-hamster ovary cells [10]. However, these currently available strategies 
for interferon- $\gamma$ acquisition somewhat limit application owing to their complicated operation procedures, higher cost, sophisticated equipment, and low production efficiency and activity. Although the most conventional model in industrial production of overexpressing recombinant human interferon- $\gamma$ is in $E$. coli, the cytoplasmic inclusion bodies and endotoxin secreted by the bacteria have been found to accumulate in the compound, making interferon- $\gamma$ production frequently subject to substandard protein purity and higher background protein. In particular, the formation of protein maintaining conserved structure and complex functions is affected [11, 12]. In addition, although recombinant human interferon $-\gamma$ was shown to be involved in antiproliferative [13] and apoptotic [14] effects against cancer cells, it also needs an exogenous supplement. These findings indicate that if we can target the endogenous interferon- $\gamma$ locus, reprogram the silenced epigenetic state in non-immune cells, and activate interferon- $\gamma$ expression, when we can ultimately treat cancer cells or prevent virus infection using the organism's innate genomic machinery [15].

The clustered regularly interspaced short palindromic repeat (CRISPR-Cas9), as a new genome engineering tool, can promote endogenous genome transcriptional activation efficiently [16]. To investigate whether CRISPR-Cas9-derived activation systems can activate the endogenous IFNG gene, we explored two systems (the dCas9-SunTag-VP64 and synergistic activation mediator (SAM) systems [17-19]) to target the interferon- $\gamma$ promoter. In both systems, catalytically inactive Streptococcus pyogenes (Sp) Cas9 nuclease-derived proteins, in combination with single-guide RNA (sgRNA), are frequently fused to VP64 activators (derived from four copies of the herpes virus transcriptional activation domain VP16) to activate expression of a specific gene [20]. In particular, the SAM system uses modifications to the loop regions in the guide RNA (gRNA) scaffold to allow for embedded RNA aptamers, such as those that correlate the dimerized bacteriophage coat protein MS2, which helps recruit various MS2-bound transcriptional activation domains, such as NF-kB transactivating subunit p65 and human heat shock factor 1 (HSF1) [17, 21, 22], to induce enhanced expression of targeted genes.

In the present study, we identified an optimal target region of the interferon- $\gamma$ promoter. sgRNAs were consistently achieved by targeting the transcriptional start site within a $-200 \mathrm{bp}$ to $+1 \mathrm{bp}$ window for acquiring the highest levels of activation [17]. Significantly, the interferon- $\gamma$ promoter was effectively and highly activated and resulted in interferon- $\gamma$ expression in several cell lines. In addition, the interferon- $\gamma$ induced by the SAM system could activate innate immunity and inhibit tumorigenesis. Our results provide an new avenue for cytokine gene therapy simply through choosing appropriate gRNA sequences to gain rapid induction of interferon- $\gamma$ in vivo [23].

\section{Materials and Methods}

\section{Plasmid and Vector Constructs}

Plasmids encoding NLS-dCas9-VP64 and MS2-p65-HSF1 (plasmid \#61425 and \#61426), and dCas9-24xGCN4-v4 and scFvGCN4-sfGFP-VP64 expression plasmids were obtained from Addgene (plasmid \#60910 and \#60904). The sgRNA expression plasmid was obtained from Addgene (plasmid \#61427), and the multiple sgRNA clone vector including BsmBI restriction sites facilitated the insertion of different gRNA target corresponding oligo DNAs. The lentiCRISPR-V2 plasmid was obtained from Addgene (plasmid \#52961) and the pGL3-basic plasmid was obtained from Promega. To obtain the interferon- $\gamma$ promoter luciferase reporter plasmid, the promoter fragment was amplified from peripheral blood mononuclear cells using the forward primer F (5'-CGGGGTACCGGGATTACAGGCGTGAGCCACC-3') and the reverse primer $\mathrm{R}$ (5'-CCGCTCGAGCGTAGATATTGCAGATAC TTCTGGGCTT-3'), followed by purification and digestion by KpnI and XhoI restriction enzymes and then ligation into the KpnI-XhoI clone sites of the pGL3-basic plasmid. Interferon- $\gamma$ promoter reporter lacking the sgRNA target site ( $\Delta$ sgRNA9 and sgRNA10)luc was constructed using two-step fusion PCR: forward primer (5'-CGGGGTACCGGGATTACAGGCGTGAGCCACC-3') and reverse primer (5'-CGTATTTTCACAAGTTTTTTAATGATAGTTTGTAT-3') amplify upstream of the $5^{\prime}$-promoter; forward primer (5'-TTA TTAATACAAACTATCATTAAAAAACTTGTG- $\left.3^{\prime}\right)$ and reverse primer (5'-CCGCTCGAGCGTAGATATTGCAGATACTTCTGGGCTT$3^{\prime}$ ) amplify downstream of the 5'-promoter. Finally, a full-length interferon- $\gamma$ lacking the sgRNA9 and sgRNA10 target site fragments was amplified using a forward primer (5'-CGGGGTACCGGG ATTACAGGCGTGAGCCACC-3') and a reverse primer (5'-CCG CTCGAGCGTAGATATTGCAGATACTTCTGGGCTT-3') with two fragments amplified in the first step as templates. The purified interferon- $\gamma$ promoter ( $\Delta$ sgRNA9 and sgRNA10) PCR product was then ligated into the KpnI- and XhoI-digested pGL3-basic plasmid. All the expression plasmids were confirmed by sequencing.

\section{Cell Culture and Transfection}

HEK293T and HeLa cells were maintained in Dulbecco's modified Eagle's medium supplemented with $10 \%$ FBS, $100 \mathrm{U}$ penicillin $\mathrm{ml}^{-1}$ and $100 \mathrm{mg}$ streptomycin $\mathrm{ml}^{-1}$ at $37^{\circ} \mathrm{C}$ and $5 \% \mathrm{CO}_{2}$. Jurkat $\mathrm{T}$ cells were maintained in RPMI 1640 medium supplemented with $10 \% \mathrm{FBS}$ at $37^{\circ} \mathrm{C}$ and $5 \% \mathrm{CO}_{2}$. HEK293T cells and HeLa cells were transfected with polyethyleneimine (PEI) and lipofectamine 2000 (Invitrogen, USA), respectively according to the manufacturer's instructions. The NLS-dCas9-VP64 and MS2-p65-HSF1 expression plasmids and individual sgRNA expression plasmid were transfected into target cells at a mass ratio of 1:1:2. The dCas9- 
24xGCN4-v4 and scFv-GCN4-sfGFP-VP64 expression plasmids and the individual sgRNA expression plasmid were also transfected at a mass ratio of 1:1:2. The NLS-dCas9-VP64 and MS2-p65-HSF1 expression plasmids and individual sgRNA expression plasmid were packaged into the lentivirus according to the standard operating procedure [24] and the Jurkat $\mathrm{T}$ cells were transduced with lentivirus at an MOI of 40 .

\section{Luciferase Reporter Assay}

To examine the effects of the SAM system or dCas9-SunTagVP64 with each gRNA on the interferon- $\gamma$ promoter, NLS-dCas9VP64, MS2-p65-HSF1, or dCas9-SunTag-VP64 (100 ng) with indicated sgRNA (200 ng) were co-transfected into HEK293T cells and HeLa cells with the interferon- $\gamma$ promoter luciferase reporter (50 ng) and internal control pRL-TK (10 ng) using PEI and lipofectamine 2000, respectively, according to the manufacturer's instructions. After $24 \mathrm{~h}$ transfection, the cells were harvested and lysed, and the collected supernatant was used to detect luciferase activity using the Dual-Luciferase Reporter Assay System (Promega, USA). Each experiment was performed in triplicate and repeated three times. The data were analyzed and presented as means \pm SD. The two-tailed Student $t$-test was used to calculate the significant difference. $p<0.05$ was considered statistically significant.

\section{Enzyme-Linked Immunosorbent Assay}

To test the protein level of interferon- $\gamma$ in the supernatant, the HEK293T cells were transfected with the SAM system-complex using PEI. After 36 and $72 \mathrm{~h}$ transfection, the effect of endogenous interferon- $\gamma$ gene activation was monitored by quantifying the amounts of interferon- $\gamma$ produced in the supernatant by using a human interferon- $\gamma$ ELISA kit (Neobioscience, China) according to the manufacturer's instructions.

\section{Quantitative RT-PCR}

The expression of IFNG, IRF1 and STAT1 was examined using relative quantitative RT-PCR. HEK293T cells were transfected with NLS-dCas9-VP64 or MS2-p65-HSF1, and sgRNA4, sgRNA9, or sgRNA10, respectively, using PEI for $48 \mathrm{~h}$. Jurkat T cells were non-transduced or transduced with SAM-sgRNA4, sgRNA9, or sgRNA10 lentivirus after 7 days of infection, and the total RNA was isolated from cells with TRIzol reagent. The isolated RNA was then reverse transcribed into cDNA using oligo(dT)12-18 primer and M-MLV reverse transcriptase (Promega) according to the manufacturer's instructions. The SYBR Green master mix (Roche Diagnostics, USA) was used for real-time PCR [25]. The target gene expression was normalized to the expression of the glyceraldehyde-3-phosphate dehydrogenase gene (GAPDH). GAPDH mRNA was analyzed to serve as the internal control. The primers used for the investigated genes are listed in Table S2. Relative fold changes in gene expression were determined using the threshold cycle $(2-\Delta \Delta \mathrm{Ct})$ method. The data were analyzed by the same statistical method as used for the luciferase reporter assay.

\section{Western Blot Assay}

HEK293T and HeLa cells were washed once with PBS, and lysed in RIPA buffer (50 mM Tris, pH 7.6, 1\% NP-40, $140 \mathrm{mM} \mathrm{NaCl}$, $0.1 \%$ SDS) and PhosSTOP (Roche, USA). Then, the protein samples were resolved by $12 \%$ SDS-polyacrylamide gel electrophoresis (PAGE) and transferred onto a nitrocellulose membrane (GE Healthcare, USA). The membrane was blocked with PBS containing $0.1 \%$ Tween 20 and 5\% skim milk and probed with antibodies against phosphorylated STAT1 (pY701-STAT1) and STAT1 (Cell Signal Technology, USA).

\section{On-Target and Off-Target Analysis Induced by CRISPR-Cas9}

On-target was measured by the CRISPR-Cas9 system with sgRNA9. The forward primer F (5'- CGGGGTACCGGGATTACA GGCGTGAGCCACC-3') and the reverse primer R (5'-CCGCTC GAGCGTAGATATTGCAGATACTTCTGGGCTT-3') were used for amplifying on-target sites in mock cells or cells transfected with Cas9 and the indicated sgRNA9, sgRNA10, or sgRNA vector. The fragment was amplified, melted, and annealed to form heteroduplex DNA. The DNA was treated with five units $(10 \mathrm{U} / \mu \mathrm{l})$ of the mismatch-sensitive T7 Endonuclease 1 (T7EN1; New England BioLabs, USA) for $30 \mathrm{~min}$ at $37^{\circ} \mathrm{C}$ and then precipitated by addition of $2 \mu \mathrm{l}$ of $0.25 \mathrm{M}$ EDTA solution. The precipitated DNA was analyzed by agarose gel electrophoresis.

Off-target analysis was performed using bioinformatics-based search tools to determine the potential off-target sites in the human genome by CRISPR-Cas9 with sgRNA9. Six potential off-target sites were identified by BLAST search in the NCBI database of the human genome and http:/ / crispr.mit.edu. The primers (Table S4) for amplifying the six off-target sites resulted in 700-800 bp amplicons in cells untreated or transfected with Cas9 and sgRNA9. Mismatchsensitive T7EN1 assay was used to detect the off-target effect.

\section{Flow Cytometry Apoptosis Analysis}

HeLa cells were transfected with the SAM system-complex using lipofectamine 2000. The interferon- $\gamma$ neutralizing antibody (R\&D System, China) was joined after $8 \mathrm{~h}$ transfection. Three days later, cells were collected with $0.25 \%$ trypsin-EDTA, rinsed with pre-cooled PBS, and then resuspended in binding buffer. Apoptosis was analyzed using the Annexin V-FITC apoptosis detection kit (BD Biosciences, US) according to the manufacturer's instructions. Cells were then incubated for $15 \mathrm{~min}$ at room temperature in dark condition. Subsequently, they were detected with the flow cytometer and analyzed with Flow Jo software.

\section{Cell Proliferation Assay.}

Cells co-transfected with the customized SAM-sgRNA system and cells joined with interferon- $\gamma$ neutralizing antibody after $8 \mathrm{~h}$ transfection were simultaneously plated onto a 96-well plate at a density of 4,000 cells per well in $100 \mu \mathrm{l}$ of fresh minimum essential medium containing $10 \%$ fetal bovine serum and incubated in an atmosphere containing $5 \% \mathrm{CO}_{2}$. Cell proliferation of these treatment 
groups was evaluated 24,48 , or $72 \mathrm{~h}$ later using the cell counting kit-8 (Beyotime Institute of Biotechnology, China) reduction assay. Briefly, $10 \mu \mathrm{l}$ of CCK-8 was added to each well and incubated for $1 \mathrm{~h}$ at $37^{\circ} \mathrm{C}$ in the dark. The production of blue formazan crystals was determined by spectrophotometric measurement of absorbance values at $450 \mathrm{~nm}$.

Carboxyfluorescein Succinimidyl Amino Ester (CFSE) Distribution Assay by Flow Cytometry

Exponentially growing HeLa cells were washed twice with FBSfree medium at $37^{\circ} \mathrm{C}$. Then the cells were resuspended in FBS-free culture medium with CFDA-SE stock solution $(10 \mu \mathrm{M})$ at $37^{\circ} \mathrm{C}$ for $10 \mathrm{~min}$ in the dark. Next, the cells were washed twice with four volumes of the culture medium and incubated in the fresh medium at $37^{\circ} \mathrm{C}$ for $5 \mathrm{~min}$ in the dark. After that, the cells were centrifuged and cultured overnight. After the cells were transfected by SAM components with sgRNA9, sgRNA10, or sgRNA vector, the fluorescence channels FL1 test was performed for detecting the effect of CFSE staining. For detecting the fluorescence of the dry-stained cells, the cells were trypsinized, resuspended in $1 \mathrm{ml}$ PBS, and then monitored using the flow cytometer after 3 or 7 days of transfection.

\section{Tumor Transplantation in Nude Mice}

All animal experiments were performed in compliance with the National Institutes of Health guidelines for the care and use of laboratory animals. Female BALB/c NOD-SCID mice (4-6 weeks of age) were purchased from the Animal Service Center of Charles River Company. Animal handling and experimental procedures were approved by the Animal Experimental Ethics Committee of Wuhan University (No. WDSKY0201502). A total of $2.0 \times 10^{7}$ Hela cells transfected by sgRNA9, sgRNA10, or sgRNA vector with SAM components were injected subcutaneously into the upper right flank near the back of nude mice $(N=6)$. Tumor volumes were measured every 2 days. The tumor volume was calculated by the equation (volume $=a \times b^{2} / 2$, where $a$ is the length and $b$ is the width of the tumor diameter) as an ellipsoid approximation. Three weeks later, the mice were euthanized and tumors were dissected and weighed. Data are represented as means \pm SD.

\section{Statistical Analysis}

Statistical analysis was performed using SPSS13.0 for Windows. Comparisons between the two groups were analyzed by paired Student's $t$-tests. Comparisons among groups were made by oneway ANOVA test. Data are presented as means \pm standard deviation of independent experiments. At least three replicates were performed for each experimental condition.

\section{Results}

Design and Screening of Optimal sgRNAs for dCas9SunTag-VP64 and SAM Systems to Activate Interferon- $\gamma$ Gene Transcription

To investigate whether the CRISPR-dCas9 system functions as a transcriptional induction tool and induces interferon- $\gamma$ expression, we explored the canonical reported SAM system and dCas9-SunTag-VP64 system, together with designed sgRNAs targeting the interferon- $\gamma$ promoter start site sequence within $200 \mathrm{bp}$ to modulate gene transcription. The SAM system (Fig. 1A) comprises three components: dCas9 [26] fused with VP64 activation domain, modified gRNA containing aptamers, and MS2 bacteriophage coat protein fused with p65 and HSF1 activation domain [17]. In the dCas9-SunTag-VP64 system (Fig. 1B), synthetic transcriptional activator VP64 is fused to single-chain variable fragment $(\mathrm{scFv})$ antibodies with binding specificity for peptides derived from the general control protein 4 (GCN4). Co-expression of these antibodies together with dCas9 fused to GCN4-containing peptide tags (Sun-Tag) and gRNAs leads to spatial recruitment of multiple VP64 domains to the gRNA-complementary target sites [18].

To design and screen optimal sgRNAs for the SAM system and dCas9-SunTag-VP64 system, we chose 10 sgRNAs (sgRNA1-10) to target the interferon- $\gamma$ promoter and tested their transactivation activity in human embryonic kidney 293T (HEK293T) cells (Table S1). Furthermore, according to NGG Streptococcus pyogenes Cas9 photospacer adjacent motif (PAM) sites, we designed gRNAs that target the upstream region of the interferon- $\gamma$ transcriptional start site ( -200 to $0 \mathrm{bp}$ ) of the genome (Fig. 1C). We co-transfected HEK293T cells with the SAM (Fig. 1D) and dCas9-SunTag-VP64 (Fig. 1E) component expression vectors, with individual sgRNAs and the interferon- $\gamma$ promoter reporter. The luciferase assays showed strong activation of reporter gene expression when targeting with each sgRNA, and sgRNA10 reached the highest level, up to 800-fold compared with the control in the SAM system (Fig. 1D). The dCas9-SunTagVP64-mediated activation results showed that the level of activation was generally lower than the SAM system, with a maximum of 150-fold activation compared with controls (sgRNA9) (Fig. 1E). Through comparison of the two systems, sgRNA4, sgRNA9, and sgRNA10 showed similar activation tendencies. Two sgRNAs (sgRNA9 and sgRNA10), complementary to the sequence $97 \mathrm{bp}$ to $71 \mathrm{bp}$ upstream of the transcription start sites, induced significant activation with the SAM system and were selected for further experimentation.

\section{Interferon- $\gamma$ Induced by the SAM System Positively Modulates Its Triggered Signaling Pathway and Enhances Relevant Downstream Gene Expression}

Biological functions of interferon- $\gamma$ are initiated depending on the integrity of their cognate receptors and activation of 
A
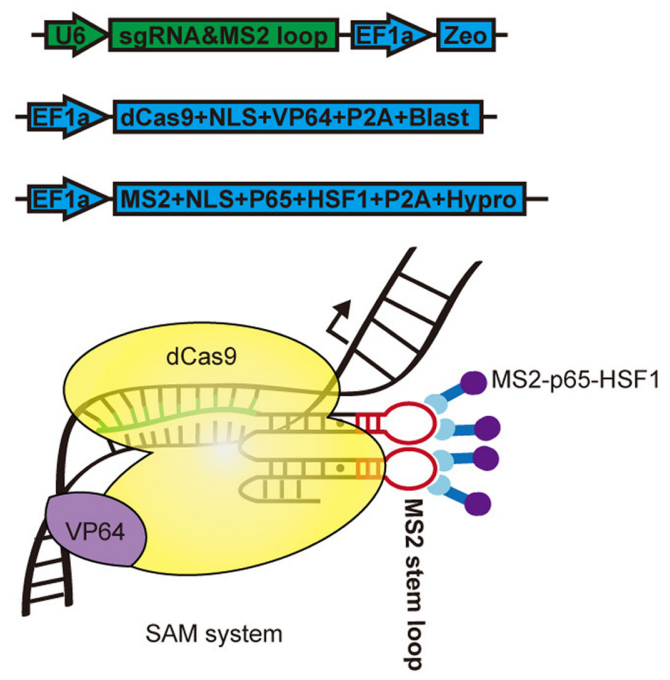

\section{B}
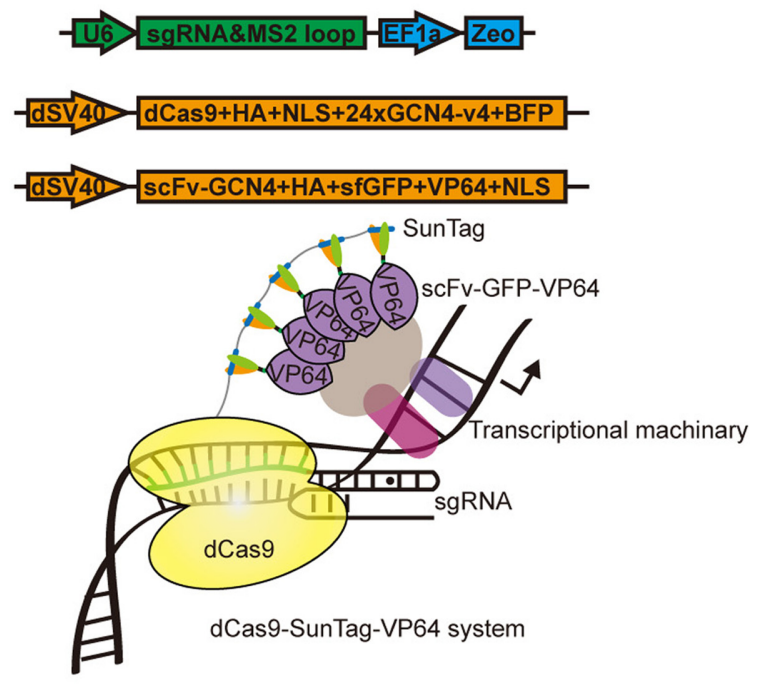

C

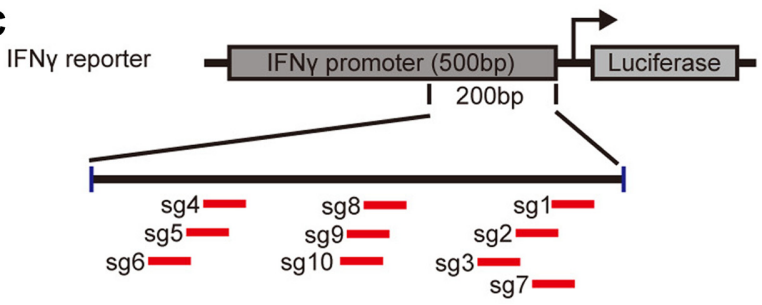

D

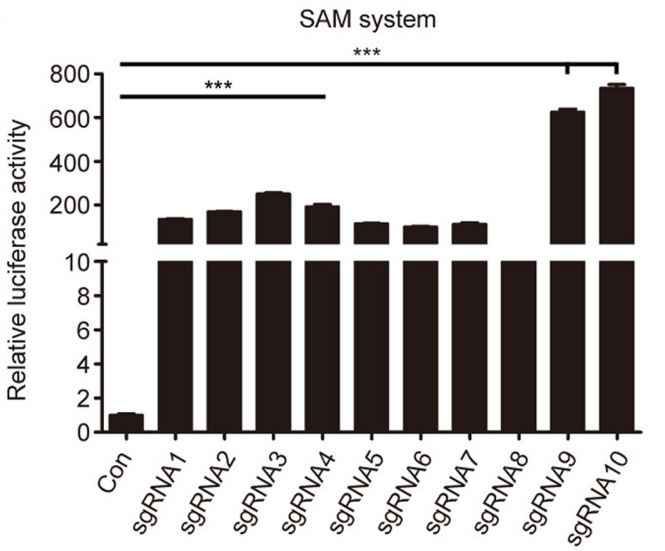

E

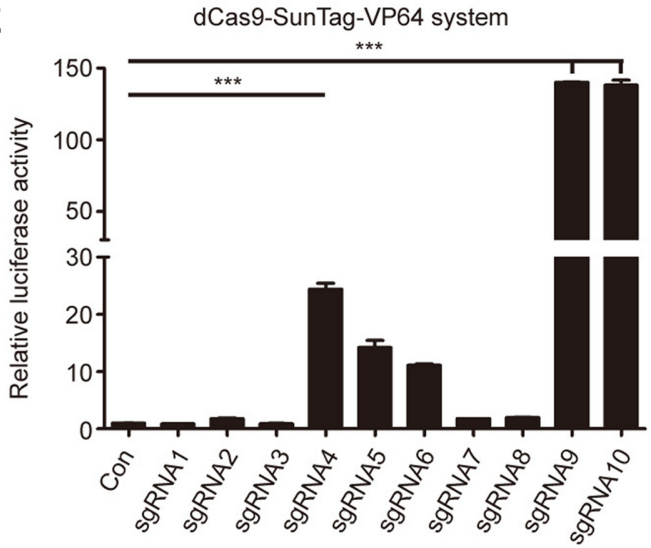

Fig. 1. Design and screening of gRNAs for the SAM and dCas9-SunTag-VP64 systems to activate interferon- $\gamma$ transcription. (A, B) Schematic description of SAM and dCas9-SunTag-VP64 activation systems. In both systems, a single-guide RNA (sgRNA) binding to a specific targeted promoter region results in recruitment of multiple activator domains of transcription factors such as VP64, HSF1, p65 etc. (C) Scheme of 10 customized gRNAs targeting the $200 \mathrm{bp}$ region upstream of the transcriptional start site (interferon- $\gamma$ promoter). (D, E) Screen of the designed complimentary sgRNAs for SAM or dCas9-SunTag-VP64 mediated interferon- $\gamma$ activation by dual-luciferase assay. HEK293T $\left(1.0 \times 10^{5}\right)$ cells were transfected with interferon- $\gamma$ reporter, PRL-TK (as an internal control), and the SAM or dCas9-SunTag-VP64 components with sgRNA1 10. Two days later, the relative luciferase activity was measured using the dual-luciferase reporter assay system. The data were analyzed by normalizing the individual sgRNA empty vector to the sgRNA-transfected group in both systems. Each data represents the means \pm SD of three independent experiments. ${ }^{* * *} p<0.001$; paired $t$-test.

the JAK-STAT signaling pathway [27]. Tyrosine phosphorylation occurs after the activation and contact of Janus kinase, which gradually in turn attracts STAT1 with an SH2binding domain into the interferon- $\gamma$ receptor and leads to the phosphorylation of STAT1 [28]. To investigate whether CRISPR-dCas9-derived activation systems could induce interferon- $\gamma$ expression and activate downstream signaling pathways, HEK293T cells were transiently co-transfected 
with components of the SAM system plus sgRNA4, sgRNA9, or sgRNA10, based on their superior activating effects, as seen in the luciferase assay (Fig. 1D). ELISA of cell culture supernatant results showed that SAM components with
sgRNA9 strongly activated interferon- $\gamma$ expression in HEK293T cells. The interferon- $\gamma$ in sgRNA9-induced cells reached up to 90 -fold over controls at $72 \mathrm{~h}$ post-transfection (Fig. 2A). We observed that transient expression of SAM
A

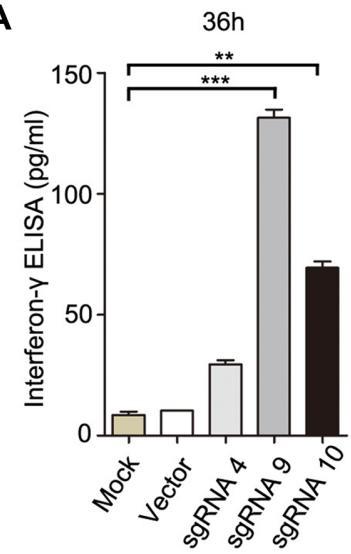

C
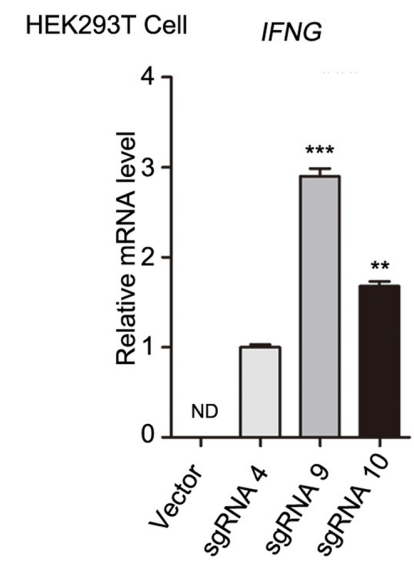

E

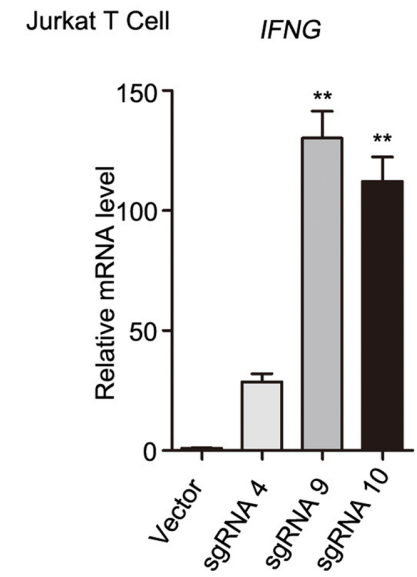

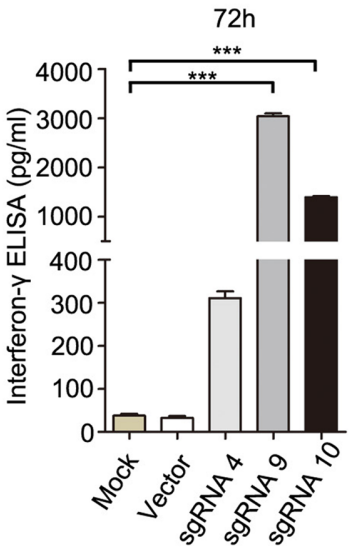

D
B

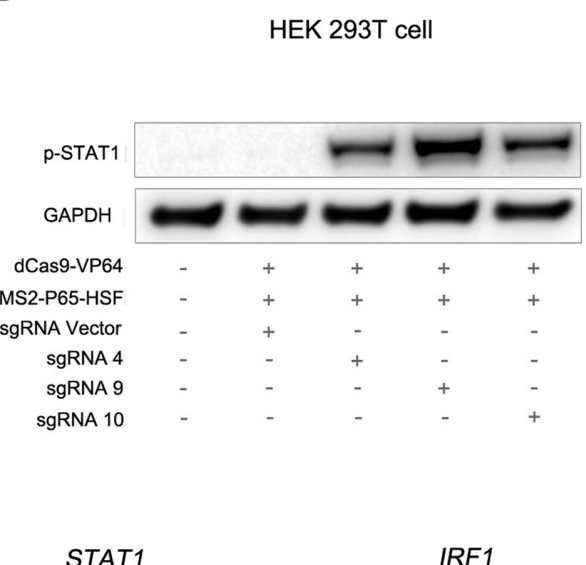

STAT1

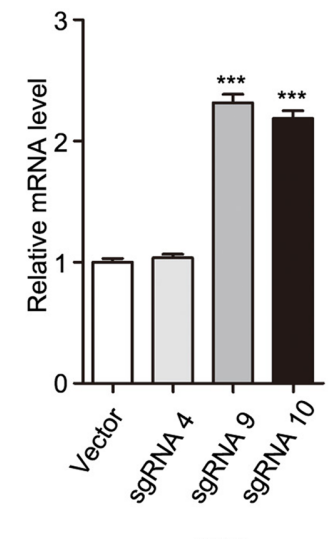

$\mathbf{F}$

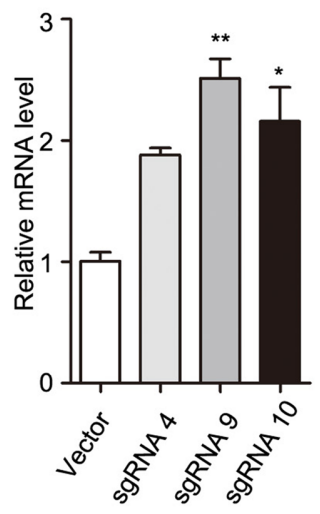

STAT1

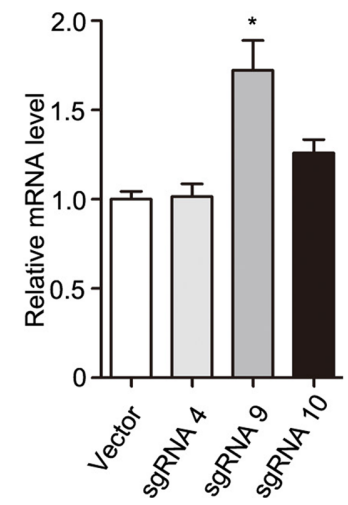

Fig. 2. Activation of the interferon- $\gamma$ gene induced through the SAM system positively regulates the JAK-STAT signaling pathway. (A) ELISA. ELISA analysis of the interferon- $\gamma$ protein level after HEK293T cells $\left(1.0 \times 10^{5}\right)$ were transiently transfected with sgRNA4, sgRNA9, sgRNA10, or sgRNA empty vector, with SAM components for 24 and $72 \mathrm{~h}$. (B) Western blotting was used to analyze p-STAT1 expression triggered by individual interferon- $\gamma$ in the cell lysates after $36 \mathrm{~h}$ transfection. qRT-PCR analysis of IFNG and downstream genes STAT1, IRF1, and GAPDH mRNA as measured after $48 \mathrm{~h}$ post-transfection in HEK293T cells (C, D) and Jurkat T cells (E, F). The values are presented as the means \pm SD of three independent experiments; ${ }^{*} p<0.05 ;{ }^{* *} p<0.01 ;{ }^{* * *} p<0.001$. 
together with optimal sgRNAs induced strong expression of interferon- $\gamma$. The phosphorylation level of STAT1 was measured using western blotting (Fig. 2B). The HEK293T cells treated with the SAM component with sgRNA4, sgRNA9, and sgRNA10 induced phosphorylation of STAT1, whereas the untreated cell and control cells (treated with gRNA empty vector) did not demonstrate induction of IFN/STAT 1 phosphorylation. We also investigated the induction effect of the SAM system on IFNG mRNA levels. As expected, IFNG mRNA was significantly enhanced in induced cells, whereas IFNG mRNA did not increase in the transiently transfected sgRNA empty vector samples (Fig. 2C).

The process by which interferon- $\gamma$ regulates the immune response to prevent infection from exogenous viruses and intracellular pathogens depends on the binding of STAT1 to the IRF1 promoter in host cells [29]. To determine whether interferon- $\gamma$ induced by the SAM system regulates downstream gene transcription, STAT1 and IRF1 mRNAs were assessed via qRT-PCR. The results demonstrated that interferon- $\gamma$ positively regulates the mRNA synthesis of IRF1 and STAT1 (Figs 2D).

Similarly, qRT-PCR results showed that the IFNG, IRF1, and STAT1 mRNA expression levels were detectable in Jurkat $\mathrm{T}$ cells, suggesting that the JAK-STAT signaling pathway could function in the T cell line (Figs. 2E and $2 F$ ).

\section{dCas9 Specifically Binds to a Designed Region of Interferon- $\gamma$ Promoter to Activate Gene Transcription}

Having demonstrated that SAM components together with optimal sgRNAs can specifically activate the interferon- $\gamma$ promoter, our results prompted the possibility of using this activation system for interferon- $\gamma$ production or employing it for clinical cytokine gene therapy. The specificity of the on-target effect is a concern in clinical therapy [30]. To test whether the SAM system activates interferon- $\gamma$ expression through direct binding to the corresponding region of promoter, we constructed an interferon- $\gamma$ promoter luciferase reporter vector lacking the designed sgRNA9 and sgRNA10 target sites simultaneously, termed interferon- $\gamma$ ( $\Delta$ sgRNA) reporter (Fig. 3A). The luciferase reporter assays demonstrated intense induction of luciferase expression from the interferon- $\gamma$ promoter reporter, but failed to induce the interferon- $\gamma$ promoter ( $\Delta$ sgRNA) reporter (Fig. 3B). In order to confirm the specificity, we took advantage of the CRISPR-Cas9 system to reconstruct a gene-editing system and used T7EN1 assays to probe for ablation of the interferon- $\gamma$ promoter. The result showed that sgRNA9 and sgRNA10 can edit the interferon- $\gamma$ promoter, indicating that dCas9 in the SAM system is specifically binding to the designed region of the promoter (Fig. 3C).

Targeted gene transcription activation using the CRISPRdCas9 system has potential off-target effects; therefore, we needed to carry out additional verification to explore this. We aligned the sgRNA9 target sequence to the human genome and searched for all the potential off-target sites using an online tool (http://crispr.mit.edu) [31]. We identified six potential off-target sites with high scores for sgRNA9 (Table S3). CRISPR-Cas9 gene editing together with T7EN1 assay was used to identify the off-target effects [32]. Our results showed that no mutation was detectable by T7EN1 analysis, suggesting that there is no off-target mutagenesis in our sgRNA transfection experiment (Fig. 3D).

\section{Interferon- $\gamma$ Induced by the SAM System Contributed to Apoptosis and Low Proliferation of HeLa Cells}

The antitumor mechanism of interferon- $\gamma$ is associated with increased apoptosis, inhibition of cell growth, and delayed growth of the tumor cell cycle [33, 34], and all these are inseparable from the integrity of interferon- $\gamma$ function. To probe whether interferon- $\gamma$ transcription activation plays a role in cancer cells, we transiently transfected the SAM component with sgRNAs into HeLa cells. The luciferase assays showed sgRNA9 and sgRNA10 reached the highest level, up to 100-fold compared with the control (Fig. 4A). Western blotting analysis showed that endogenous interferon- $\gamma$ can promote the phosphorylation of STAT1 efficiently (Fig. 4B). These data demonstrate that transcriptional activation of the endogenous interferon- $\gamma$ gene in cancer cells similarly promotes interferon- $\gamma$-triggered STAT1 phosphorylation [35].

To probe for the apoptosis and inhibition of cell growth by interferon- $\gamma$, apoptosis and CCK-8 assays were carried out and the result shows that they, including sgRNA9 and sgRNA10, could induced cell apoptosis and inhibit cell proliferation (Figs. 4C and 4D). To test whether such effects were interferon- $\gamma$-dependent, interferon- $\gamma$ neutralizing $\mathrm{Ab}$ was joined for comparison in cell apoptosis and proliferation assays with the same method, and showed no obvious differences both in apoptosis rate and cell proliferation (Figs. 4C and 4D). Next, CFSE distribution assays were carried out to monitor long-term cell proliferation [36]. As the cells proliferate and divide, parental cells, marked by carboxyfluorescein (CF) fluorescence, evenly distribute CF to the daughter cells. Therefore, the fluorescent intensities weaken as the cells divide. Using this method, the HeLa 
A

IFNy pro TAATGGGTCTGTCTCATCGTCAAAGGACCCAAGGAGTCTA IFNy pro TAATGGGTCTGTCTCATCGTCAAAGGACCCAAGGAGTCTA $(\triangle \mathrm{sgRNA})$

AAGGAAACTCTAACTACAACACCCAAATGCCACAAAACCT AAGGAAACTCTAACTACAACACCCAAATGCCACAAAACCT

TAGTTATTAATACAAACTATCATCССTGCCTATCTGTCAC TAGTTATTAATACAAACTATCAT-

САТСТСАТСТTАAАAАACTTGTGAAAATACGTAATCCTCA --------TAAAAAACTTGTGAAAATACGTAATCCTCA GGAGACTTCAATTAGGTATAAATACCAGCAGCCAGAGGAG GGAGACTTCAATTAGGTATAAATACCAGCAGCCAGAGGAG

C

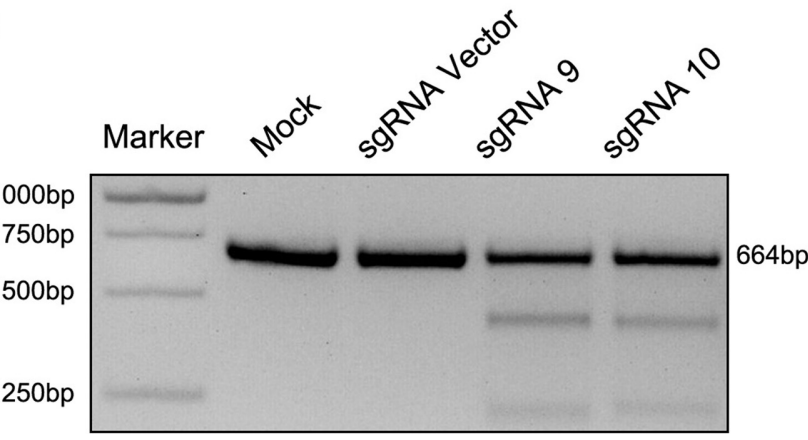

B

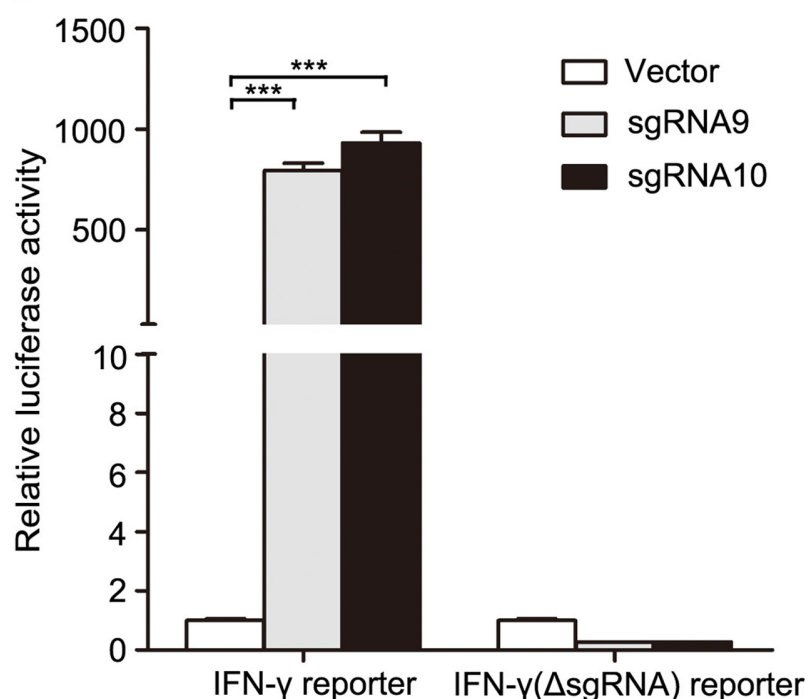

D
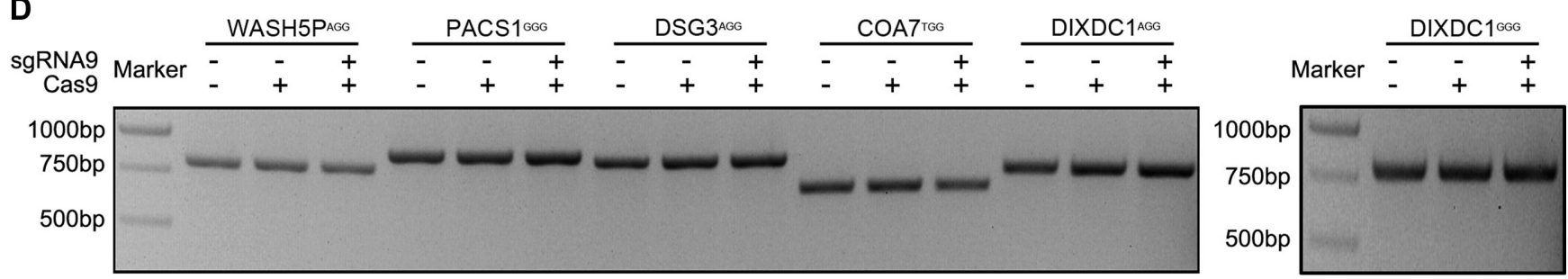

Fig. 3. dCas9 mediated by gRNA specifically binds to the interferon- $\gamma$ promoter to activate gene transcription.

(A) Schematic representation of the deletion of the sgRNA9 and sgRNA10 targeted sites in the interferon- $\gamma$ promoter luciferase reporter aligned with wild-type interferon- $\gamma$ promoter luciferase vector. (B) Co-transfection of SAM components with sgRNA9, sgRNA10, or sgRNA vector and the interferon- $\gamma$ promoter or IFN- $\gamma$ promoter $\left(\Delta\right.$ sgRNA9 and 10) reporter plasmids into HEK293T cells $\left(1.0 \times 10^{5}\right)$ at the same time. The luciferase activity induced by SAM complex including sgRNA9 or sgRNA10 was measured and normalized to that of sgRNA vector in each group at $48 \mathrm{~h}$ post-transfection. The data represent the mean \pm SD of three independent experiments; ${ }^{* * *} p<0.001$; paired $t$-test. (C) T7EN1 assay of sgRNA9 and sgRNA10-lentiCRISPR/Cas9 targeting interferon- $\gamma$ promoter in HEK293T cells. HEK293T cells $\left(1.0 \times 10^{5}\right)$ were non-transfected or transfected with sgRNA9 or sgRNA10 constructed into the lentiCRISPR/Cas9 expression vector. After $48 \mathrm{~h}$ transfection, the genomic DNA was extracted and PCR amplified for T7EN1 assay. The lower bands indicated the disrupted target alleles. (D) Off-target analysis for dCas9/sgRNA9. The off-target sites were predicted and aligned with the human genome. Six potential off-target sites were amplified from sgRNA9-lentiCRISPR/Cas9 treated cells.

cells treated with gRNA9 or gRNA10 for 3 or 7 days substantially fell behind untreated cells in proliferation rates (Fig. 4E).

\section{Interferon- $\gamma$ Induced by the SAM System Restricts Tumor Growth}

Next, we asked whether sgRNA9- or sgRNA10-induced interferon- $\gamma$ expression in tumor cells has antineoplastic bioactivity in vivo. Xenograft assays using NOD-SCID mice showed a significant reduction in the tumor volume for HeLa cells transfected with sgRNA9 and sgRNA10 compared with the vector control (Figs. 5A and 5B). In addition, the tumor growth curves showed that sgRNA9and sgRNA10-induced tumors appeared later and grew slower than control sgRNA (Fig. 5C). This significant difference in tumor formation was paralleled by similar values in the wet weight of tumors excised at the time of necropsy (Fig. 5D). Taken together, the SAM system prevents 
A

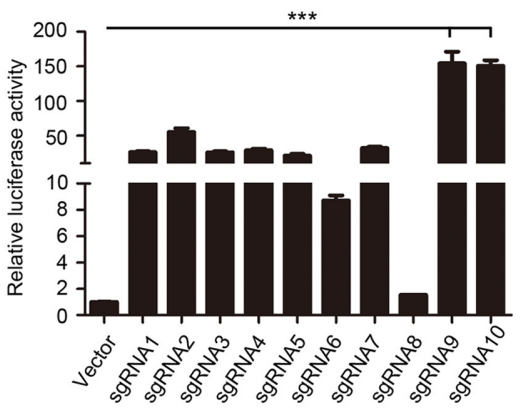

C
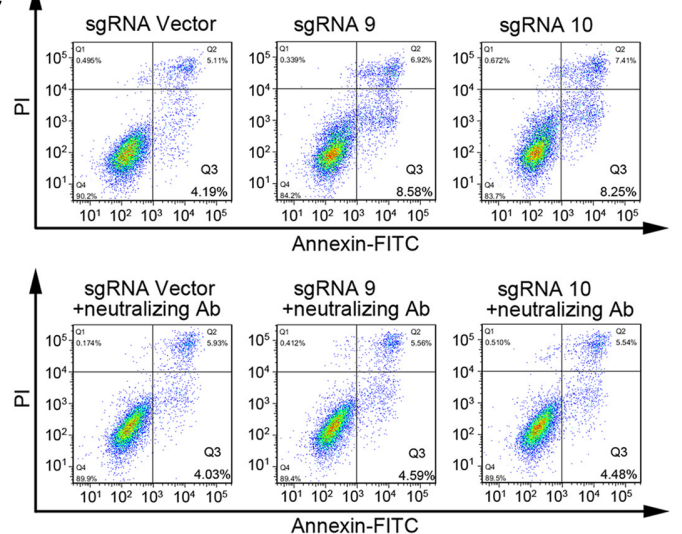

B
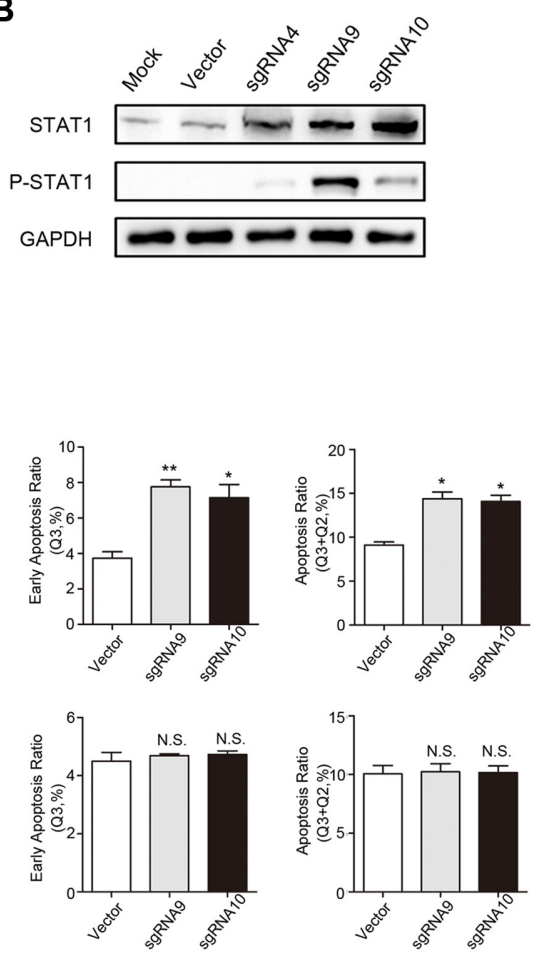

D
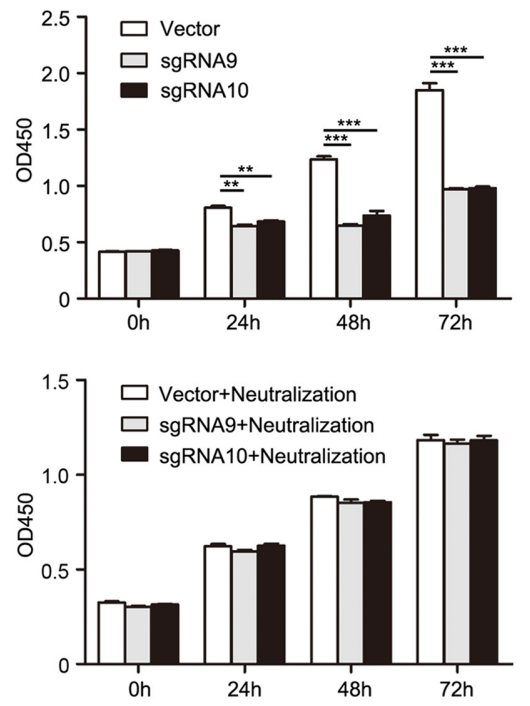

E

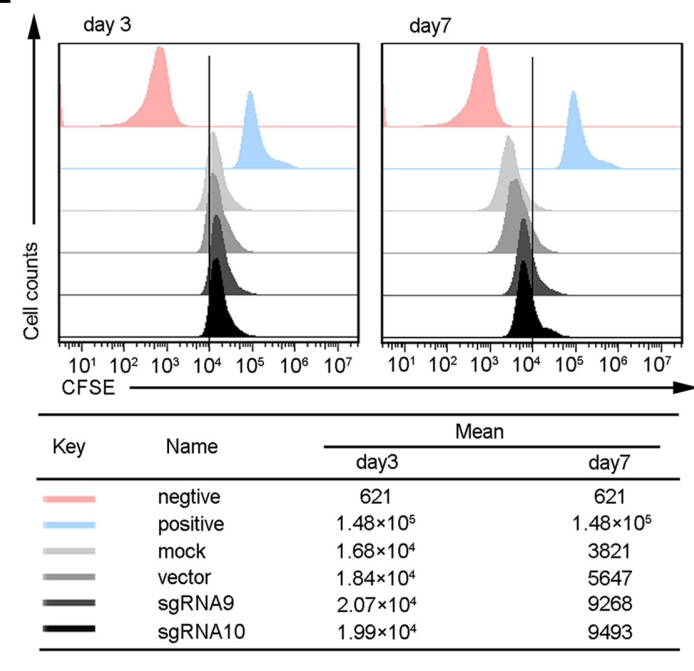

Fig. 4. Interferon- $\gamma$ induced by the SAM system triggers the STAT signaling pathway and contributes to apoptosis and low proliferation in HeLa cells.

(A) Screen of the designed complimentary sgRNAs for SAM-mediated interferon- $\gamma$ activation by dual-luciferase assay in HeLa cells. (B) Western blotting was used to analyze STAT1 and p-STAT1 expression triggered by individual interferon- $\gamma$ expression in the HeLa cells after $48 \mathrm{~h}$ transfection. (C) Apoptotic cells were determined by flow cytometry after transfection with the customized SAM system or joined interferon- $\gamma$ neutralizing $\mathrm{Ab}$ together for $72 \mathrm{~h}$. The early apoptosis ratio and total apoptosis ratio (early apoptosis and late apoptosis) were quantified by Flow J software. The data shown are the mean \pm SD of 3 independent experiments. ${ }^{*} p<0.05 ;{ }^{* *} p<0.01$; $t$-test. (D) Comparison of cell proliferation in SAM systems with and without interferon- $\gamma$ neutralizing antibody by CCK-8 assay within $72 \mathrm{~h}$ after transfection. (E) The effect of selected SAMcomplex on cell proliferation in HeLa cells was detected using the CFSE distribution assay by flow cytometry. The carboxyfluorescein fluorescence of CFSE-labeled HeLa cells $\left(1.0 \times 10^{5}\right)$ transiently transfected with SAM component with sgRNA9, sgRNA10, or sgRNA vector is shown. These cells and non- transfected CFSE-labeled HeLa cells were detected after 3 and 7 days of incubation. Negative means no-CFSE-labeled cells; Positive means cells immediately measured once CFSE-labeled. 
tumorigenesis of HeLa cells in vivo by activating interferon- $\gamma$ gene expression, which provides a new perspective for cancer therapy, through activating endogenous cytokines.

\section{Discussion}

Here, we present a proof of concept study that the CRISPR-dCas9 activation system can induce interferon- $\gamma$ expression. In particular, using the customized sgRNAs in combination with the SAM component, we achieved strong activation of interferon- $\gamma$, of which can be used in clinical research and therapy. We first identified an optimal target region for recruiting CRISPR-dCas9 activation systems to the interferon- $\gamma$ promoter and the specificity for the binding sites. Previous research showed sgRNAs that bind to the sequence $-200 \mathrm{bp}$ to $+1 \mathrm{bp}$ region upstream of the transcriptional start site can obtain stronger activation, and our data also indicated that sgRNAs complementary to sequence $97 \mathrm{bp}$ to $71 \mathrm{bp}$ upstream present the same effect
[17]. Furthermore, the possibility of achieving different activation levels depends on the types of targeted promoter (cell type or tissue-dependent promoters), and the interferon- $\gamma$ promoter in non-immune cells has been regarded as a type of inactive promoter [37]. In addition, our customized sgRNAs have evident overlap in the target region according to PAM sites, and this multiplexed targeting sgRNA may be one of the reasons leading to the high activation.

Notably, in the context of recent reports, the CRISPRdCas9 system-mediated activators only focus on studying HIV latency reversal and special epigenetic modifications $[38,39]$. Our study focused in particular on the applicability of humanized interferon- $\gamma$ in innate immunity. Here, we are the first to demonstrate that induction using the CRISPR-dCas9 system can result in activation and release of interferon- $\gamma$. Such levels of complete interferon- $\gamma$ are thought to be an essential prerequisite for antiviral or antitumor functions. The potentially significant advantages
A

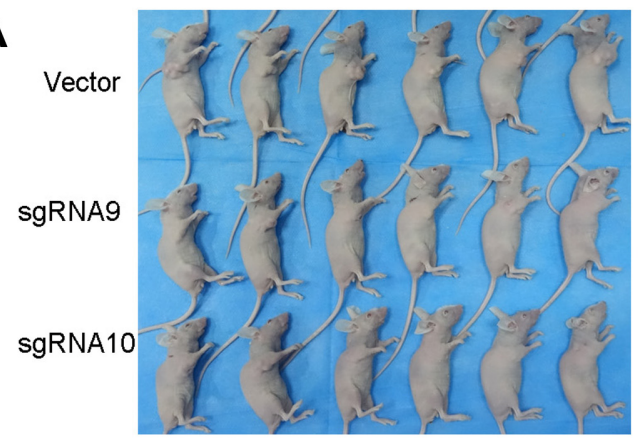

C

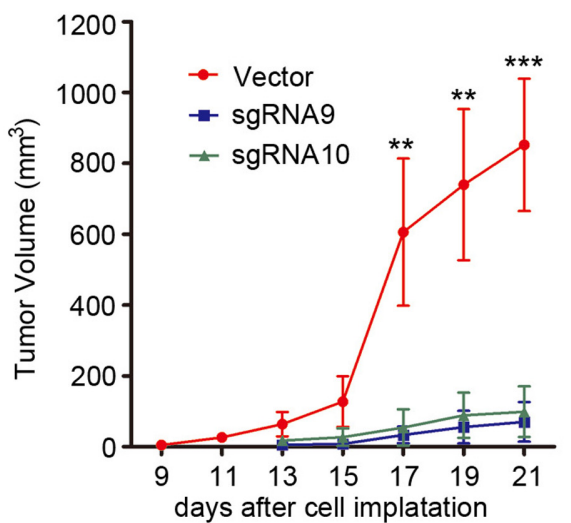

B

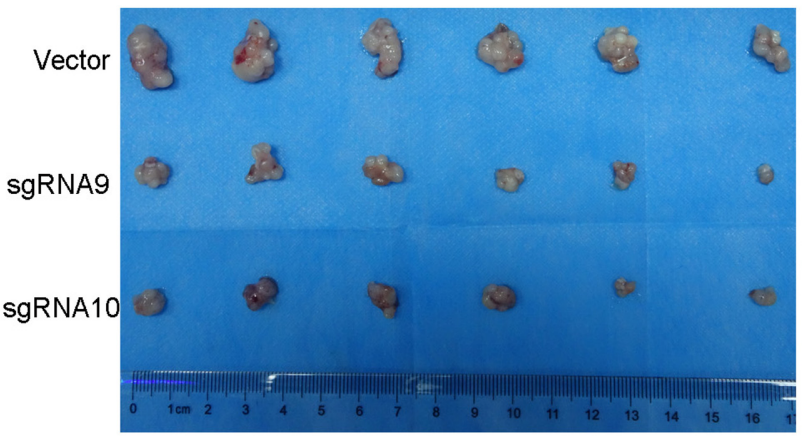

D

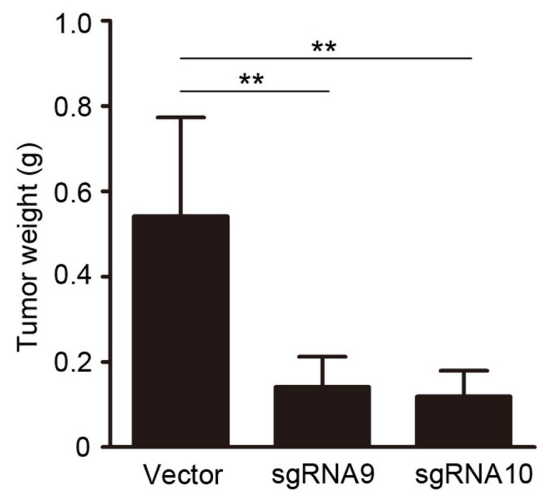

Fig. 5. Interferon- $\gamma$ induced by the SAM system inhibits transplantation tumor growth in NOD-SCID mice.

(A) Cells treated with customized SAM systems were subcutaneously injected into mice. (B) Representative gross appearance of tumors excised from NOD-SCID mice sacrificed at the end of three weeks. (C) Tumor volumes were measured every 2 days, after the eleventh day posttransplantation, tumor volumes were calculated using the equation (volume $=a \times b^{2} / 2$, where $a$ is the length and $b$ is the width of the tumor). Data are presented as means \pm SD. (D) A significant reduction in the wet weight of tumors excised at the end of the third week with designed gRNA9and gRNA10-SAM systems. All mice were euthanized and tumors were dissected and the tumor was weighed; data are presented as means \pm SD; ${ }^{*} p<0.05,{ }^{* *} p<0.01 ; t$-test. 
of inducing through transcription activation over currently used recombinant interferon- $\gamma$ are simplicity, accuracy, and humanization. The regulation of global gene expression via cellular pathways, as means of non-exogenetic therapy methods, harbor considerable potential for immature structures of recombinant interferon [17]. Since precision medicine requires tissue- and organ-targeting interferon, such fusion activators therefore have tremendous potential in targeting virus-infected cells or tumor cells to produce interferon [30]. Site-specific activation determined by sgRNAs against cell- or tissue-dependent promoters should be applied to pharmaceutical research and clinical treatment.

In the future, our current findings will be expanded to primary cells, such as chronic hepatitis patient-derived cells. A few experimental issues must be overcome prior to clinical therapy; for example, selection of effective and safe vectors to deliver customized activators [40]. The typical flow cytometric results showed that the cells treated with the SAM component in combination with sgRNA vector also delay the migration slightly; after all, undesirable gene expression in targeted cells might have potential cytotoxicity in proliferation. Another challenge for application is that once activated, potential toxicity and inflammation will influence somatic cells.

In conclusion, we have achieved a locus-specific high activator using the CRISPR-dCas9 activation machinery and unique sgRNAs targeting the interferon- $\gamma$ gene promoter. Customized sgRNAs with the SAM component resulted in increased gene expression of interferon- $\gamma$ that demonstrated normal biological activity. These findings demonstrate that activation systems based on SAM technology may provide a promising tool for the specific and targetable activation of humanized interferon- $\gamma$. The novel findings also offer an alternative therapeutic strategy for defending against viral infection and tumorigenesis via activation of functional endogenous genes.

\section{Acknowledgments}

We thank Yilin Li for technical assistance. We also thank Dr. Corine St. Gelais for revising the article. This work was supported by the Natural Science Foundation of China (81401659), China Postdoctoral Science Foundation (2015T80838 and 2014M560622), and the scholarship from the China Scholarship Council. The work was partially funded by the China National Special Research Program of Major Infectious Diseases (2014ZX10001003) and Hubei Provincial Science \& Technology Innovation Team Grant (\#2012FFA043). We are grateful to Dr. Ying Hu for the confocal assay with Wuhan University Experiment Technology Project Funding (WHU-2014-SYJS-02/204610400022).

\section{References}

1. Nandre RM, Jawale CV, Lee JH. 2013. Enhanced protective immune responses against Salmonella Enteritidis infection by Salmonella secreting an Escherichia coli heat-labile enterotoxin B subunit protein. Comp. Immunol. Microbiol. Infect. Dis. 36: 537-548.

2. Sadler AJ, Williams BR. 2008. Interferon-inducible antiviral effectors. Nat. Rev. Immunol. 8: 559-568.

3. Giroux M, Schmidt M, Descoteaux A. 2003. IFN-gammainduced MHC class II expression: transactivation of class II transactivator promoter IV by IFN regulatory factor- 1 is regulated by protein kinase C-alpha. J. Immunol. 171: 41874194.

4. Zaidi MR, Merlino G. 2011. The two faces of interferongamma in cancer. Clin. Cancer Res. 17: 6118-6124.

5. Mohammadian-Mosaabadi J, Naderi-Manesh H, Maghsoudi N, Nassiri-Khalili MA, Masoumian MR, Malek-Sabet N. 2007. Improving purification of recombinant human interferon gamma expressed in Escherichia coli; effect of removal of impurity on the process yield. Protein Expr. Purif. 51: 147-156.

6. Arakawa T, Hsu YR, Yphantis DA. 1987. Acid unfolding and self-association of recombinant Escherichia coli derived human interferon-gamma. Biochemistry 26: 5428-5432.

7. Fieschko JC, Egan KM, Ritch T, Koski RA, Jones M, Bitter GA. 1987. Controlled expression and purification of human immune interferon from high-cell-density fermentations of Saccharomyces cerevisiae. Biotechnol. Bioeng. 29: 1113-1121.

8. Ghosalkar A, Sahai V, Srivastava A. 2008. Secretory expression of interferon-alpha $2 \mathrm{~b}$ in recombinant Pichia pastoris using three different secretion signals. Protein Expr. Purif. 60: 103-109.

9. Chen YJ, Chen WS, Wu TY. 2005. Development of a bicistronic baculovirus expression vector by the Rhopalosiphum padi virus 5 ' internal ribosome entry site. Biochem. Biophys. Res. Commun. 335: 616-623.

10. Tan HK, Lee MM, Yap MG, Wang DI. 2008. Overexpression of cold-inducible RNA-binding protein increases interferongamma production in Chinese-hamster ovary cells. Biotechnol. Appl. Biochem. 49: 247-257.

11. Reddy PK, Reddy SG, Narala VR, Majee SS, Konda S, Gunwar S, et al. 2007. Increased yield of high purity recombinant human interferon-gamma utilizing reversed phase column chromatography. Protein Expr. Purif. 52: 123-130.

12. Petrov S, Nacheva G, Ivanov I. 2010. Purification and refolding of recombinant human interferon-gamma in ureaammonium chloride solution. Protein Expr. Purif. 73: 70-73.

13. Chin YE, Kitagawa M, Su W-C, You Z-H. 1996. Cell growth arrest and induction of cyclin-dependent kinase inhibitor p21WAF1/CIP1 mediated by STAT1. Science 272: 719-722.

14. Chawla-Sarkar M, Lindner D, Liu Y-F, Williams B, Sen G, 
Silverman R, et al. 2003. Apoptosis and interferons: role of interferon-stimulated genes as mediators of apoptosis. Apoptosis 8: 237-249.

15. Falahi F, Sgro A, Blancafort P. 2015. Epigenome engineering in cancer: fairytale or a realistic path to the clinic? Front. Oncol. 5: 22.

16. Doudna JA, Charpentier E. 2014. Genome editing. The new frontier of genome engineering with CRISPR-Cas9. Science 346: 1258096.

17. Konermann S, Brigham MD, Trevino AE, Joung J, Abudayyeh OO, Barcena C, et al. 2015. Genome-scale transcriptional activation by an engineered CRISPR-Cas9 complex. Nature 517: 583-588.

18. Tanenbaum ME, Gilbert LA, Qi LS, Weissman JS, Vale RD. 2014. A protein-tagging system for signal amplification in gene expression and fluorescence imaging. Cell 159: 635-646.

19. Qi LS, Larson MH, Gilbert LA, Doudna JA, Weissman JS, Arkin AP, et al. 2013. Repurposing CRISPR as an RNA-guided platform for sequence-specific control of gene expression. Cell 152: 1173-1183.

20. Shalem O, Sanjana NE, Zhang F. 2015. High-throughput functional genomics using CRISPR-Cas9. Nat. Rev. Genet. 16: 299-311.

21. van Essen D, Engist B, Natoli G, Saccani S. 2009. Two modes of transcriptional activation at native promoters by NF-кB p65. PLoS Biol. 7: e1000073.

22. Marinho HS, Real C, Cyrne L, Soares H, Antunes F. 2014. Hydrogen peroxide sensing, signaling and regulation of transcription factors. Redox Biol. 2: 535-562.

23. Naldini L. 2015. Gene therapy returns to centre stage. Nature 526: 351-360.

24. Hou P, Chen S, Wang S, Yu X, Chen Y, Jiang M, et al. 2015. Genome editing of CXCR4 by CRISPR/Cas9 confers cells resistant to HIV-1 infection. Sci. Rep. 5: 15577.

25. Paulus C, Krauss S, Nevels M. 2006. A human cytomegalovirus antagonist of type I IFN-dependent signal transducer and activator of transcription signaling. Proc. Natl. Acad. Sci. USA 103: 3840-3845.

26. Mali P, Aach J, Stranges PB, Esvelt KM, Moosburner M, Kosuri S, et al. 2013. CAS9 transcriptional activators for target specificity screening and paired nickases for cooperative genome engineering. Nat. Biotechnol. 31: 833-838.

27. Platanias LC. 2005. Mechanisms of type-I- and type-IIinterferon-mediated signalling. Nat. Rev. Immunol. 5: 375-386.

28. Schindler C, Levy DE, Decker T. 2007. JAK-STAT signaling: from interferons to cytokines. J. Biol. Chem. 282: 20059-20063.
29. Rosowski EE, Nguyen QP, Camejo A, Spooner E, Saeij JP. 2014. Toxoplasma gondii inhibits gamma interferon (IFN- $\gamma$ )and IFN- $\beta$-induced host cell STAT1 transcriptional activity by increasing the association of STAT1 with DNA. Infect. Immun. 82: 706-719.

30. Lu X, Jin X, Huang Y, Wang J, Shen J, Chu F, et al. 2014. Construction of a novel liver-targeting fusion interferon by incorporation of a Plasmodium region I-plus peptide. Biomed. Res. Int. 2014: 261631.

31. Shalem O, Sanjana NE, Hartenian E, Shi X, Scott DA, Mikkelsen TS, et al. 2014. Genome-scale CRISPR-Cas9 knockout screening in human cells. Science 343: 84-87.

32. Hsu PD, Scott DA, Weinstein JA, Ran FA, Konermann S, Agarwala V, et al. 2013. DNA targeting specificity of RNAguided Cas9 nucleases. Nat. Biotechnol. 31: 827-832.

33. Friesel R, Komoriya A, Maciag T. 1987. Inhibition of endothelial cell proliferation by gamma-interferon. J. Cell Biol. 104: 689-696.

34. Sedger LM, Shows DM, Blanton RA, Peschon JJ, Goodwin RG, Cosman D, et al. 1999. IFN- $\gamma$ mediates a novel antiviral activity through dynamic modulation of TRAIL and TRAIL receptor expression. J. Immunol. 163: 920-926.

35. Subramaniam PS, Larkin J 3rd, Mujtaba MG, Walter MR, Johnson HM. 2000. The COOH-terminal nuclear localization sequence of interferon gamma regulates STAT1 alpha nuclear translocation at an intracellular site. J. Cell Sci. 113: 2771-2781.

36. Rodel F, Franz S, Sheriff A, Gaipl U, Heyder P, Hildebrandt G, et al. 2005. The CFSE distribution assay is a powerful technique for the analysis of radiation-induced cell death and survival on a single-cell level. Strahlenther. Onkol. 181: 456-462.

37. Bikard D, Jiang W, Samai P, Hochschild A, Zhang F, Marraffini LA. 2013. Programmable repression and activation of bacterial gene expression using an engineered CRISPRCas system. Nucleic Acids Res. 41: 7429-7437.

38. Ji H, Jiang Z, Lu P, Ma L, Li C, Pan H, et al. 2016. Specific reactivation of latent HIV-1 by dCas9-SunTag-VP64-mediated guide RNA targeting the HIV-1 promoter. Mol. Ther. 24: 508-521.

39. Choudhury SR, Cui Y, Lubecka K, Stefanska B, Irudayaraj J. 2016. CRISPR-dCas9 mediated TET1 targeting for selective DNA demethylation at BRCA1 promoter. Oncotarget 7: 46545-46556.

40. Kay MA, Glorioso JC, Naldini L. 2001. Viral vectors for gene therapy: the art of turning infectious agents into vehicles of therapeutics. Nat. Med. 7: 33-40. 